\title{
VORTICAL FLOW OUTSIDE A SPHERE AND SOUND GENERATION*
}

\author{
OMAR M. KNIO ${ }^{\dagger}$ AND LU TING
}

\begin{abstract}
Formulas are presented for an incompressible inviscid velocity field $\mathbf{V}$ with a vorticity field $\boldsymbol{\Omega}$ outside of a rigid sphere and for the far-field sound generation. The velocity $\mathbf{V}$ is expressed as the sum of an image velocity $\mathbf{v}^{*}$ and a known velocity $\mathbf{v}$ in $\Re^{3}$, which is induced by the same vorticity field $\boldsymbol{\Omega}$ outside the sphere and the extension $\boldsymbol{\Omega}=0$ inside. We derive formulas for the image velocity $\mathbf{v}^{*}$ and the corresponding image potential $\Phi^{*}$, which in turn yields the far-field sound. These formulas are applied to define the velocity of a slender vortex filament in the presence of a rigid sphere and the associated far-field sound.
\end{abstract}

Key words. vortical flow, acoustic field, spherical images

AMS subject classifications. 76C05, 76Q05

PII. S003613999529397X

1. Introduction. The image of a potential flow induced by a rigid sphere was presented by Weiss in 1944 [1]. Here, we quote his results to prepare for the extension to a rotational flow. Let $\Phi_{0}(\mathbf{x})$ be a velocity potential with singularities outside the sphere $\mathcal{S}(r=|\mathbf{x}|=a)$. Then the potential solution $\Phi$ due to the presence of the sphere $\mathcal{S}$ can be written as

$$
\Phi(\mathbf{x})=\Phi_{0}(\mathbf{x})+\Phi^{*}(\mathbf{x}),
$$

where the image potential induced by the sphere $\Phi^{*}$ is related to $\Phi_{0}$ by

$$
\Phi^{*}(\mathbf{x})=\frac{a}{r} \Phi_{0}\left(\frac{a^{2} \mathbf{x}}{r^{2}}\right)-\frac{2}{a r} \int_{0}^{a} \lambda \Phi_{0}\left(\frac{\lambda^{2} \mathbf{x}}{r^{2}}\right) d \lambda .
$$

In terms of the spherical coordinates $(r, \theta, \phi)$, the image $\Phi^{*}$ takes the form given in $[2]$,

$$
\Phi^{*}(r, \theta, \phi)=\frac{1}{a} \int_{0}^{a^{2} / r} \partial_{r^{\prime}} \Phi_{0}\left(r^{\prime}, \theta, \phi\right) r^{\prime} d r^{\prime}
$$

It was shown in [1] that $\Phi^{*}$ fulfills the requirements:

$\{1\} \Phi^{*}$ is regular and harmonic for $r \geq a$.

$\{2\} \Phi^{*}=O\left(r^{-2}\right)$ for large $r$.

$\{3\} \Phi^{*}$ satisfies the boundary condition, $\partial_{r} \Phi^{*}+\partial_{r} \Phi_{0}=0$ on $r=a$.

Since $a^{2} / r<a$ for $r>a$, (2) says that

$\{4\}$ the image $\Phi^{*}$ outside the sphere depends only on the potential $\Phi_{0}$ inside the sphere.

* Received by the editors October 30, 1995; accepted for publication (in revised form) April 24, 1996.

http://www.siam.org/journals/siap/57-4/29397.html

${ }^{\dagger}$ Department of Mechanical Engineering, The Johns Hopkins University, Baltimore, MD 21218 (knio@flame.me.jhu.edu).

${ }^{\ddagger}$ Courant Institute of Mathematical Sciences, New York University, New York, NY 10012 (ting@ting.cims.nyu.edu). The research of this author was partially supported by Air Force Office of Scientific Research grant F49620-93-1-0027. 
Now we consider an incompressible inviscid flow in $\Re^{3}$ with a vorticity field $\boldsymbol{\Omega}(t, \mathbf{x})$ that vanishes inside the sphere $\mathcal{S}$ and decays exponentially in $r=|\mathbf{x}|$ in the far field. The velocity field $\mathbf{v}$ may be rotational outside of $\mathcal{S}$, where $\boldsymbol{\Omega}$ may differ from zero, with

$$
\nabla \times \mathbf{v}=\mathbf{\Omega}
$$

but is irrotational inside $\mathcal{S}$,

$$
\nabla \times \mathbf{v}=\boldsymbol{\Omega}=0 \quad \text { for } \quad r \leq a .
$$

A standard procedure is to decompose the velocity field into two parts [3],

$$
\mathbf{v}=\nabla \psi+\nabla \times \mathbf{A},
$$

where $\mathbf{A}$ denotes the divergence-free vector potential induced by $\boldsymbol{\Omega}$ and $\psi$ the background velocity potential with singularities outside $\mathcal{S}$. The background potential $\psi$ is prescribed independent of the vorticity field and its image is given by Weiss's formula (2). Therefore, it suffices to study the image of the rotational flow $\mathbf{v}$ without a background potential flow, i.e.,

$$
\psi(t, \mathbf{x})=0 \quad \text { and } \quad \mathbf{v}(t, \mathbf{x})=\nabla \times \mathbf{A}(t, \mathbf{x}) .
$$

In relating $\mathbf{v}$ to $\boldsymbol{\Omega}$, we are not dealing with the evolution of the vorticity field so we shall omit the $t$-dependency similar to what is done in [1]. Since $\Delta \mathbf{A}=-\boldsymbol{\Omega}$, the vector potential $\mathbf{A}$ is related to $\boldsymbol{\Omega}$ by the Poisson integral ${ }^{1}$

$$
\mathbf{A}(\mathbf{x})=\frac{1}{4 \pi} \iiint_{\Re^{3}} \frac{\boldsymbol{\Omega}(\mathbf{y})}{|\mathbf{x}-\mathbf{y}|} d \mathcal{V}
$$

and the velocity is related to the vorticity by

$$
\mathbf{v}(\mathbf{x})=\frac{-1}{4 \pi} \iiint_{\Re^{3}} \frac{(\mathbf{x}-\mathbf{y}) \times \mathbf{\Omega}(\mathbf{y})}{|\mathbf{x}-\mathbf{y}|^{3}} d \mathcal{V},
$$

where $d \mathcal{V}=d y_{1} d y_{2} d y_{3}$. Because of (4), the domain of integration can be reduced to $R=|\mathbf{y}| \geq a$. If $\boldsymbol{\Omega}$ is of bounded support, then the domain of integration is reduced to the support of $\boldsymbol{\Omega}$. The corresponding velocity field becomes a potential flow on and inside $\mathcal{S}$. Thus there exists a velocity potential $\Phi_{0}(\mathbf{x})$, such that $\mathbf{v}=\nabla \times \mathbf{A}=\nabla \Phi_{0}$ and $\Delta \Phi_{0}=0$, for $r \leq a$. Since $\mathbf{v}$ is defined by (6) in $\Re^{3}$, we can define $\Phi_{0}$ by the integral of the radial velocity component along the radial line $\mathbf{y}=r^{\prime} \hat{r}$ from the origin to $\mathbf{x}=r \hat{r}$

$$
\Phi_{0}(\mathbf{x})=\int_{0}^{r} d r^{\prime} \hat{r} \cdot \mathbf{v}(\mathbf{y}) \quad \text { for } \quad r \leq a .
$$

We could construct the potential $\Phi_{0}$ inside the sphere using (7), and then use Weiss's formula (2) to define the image $\Phi^{*}$ outside of the sphere. Consequently, $\Phi^{*}$ will be defined by a double integral of $\hat{r} \cdot \mathbf{v}$, which in turn is defined by a triple integral of $\boldsymbol{\Omega}$ in (6). We can then reduce this 5 -tuple integral of $\boldsymbol{\Omega}$ to a triple integral.

\footnotetext{
${ }^{1}$ The solution presumes that the velocity is continuous. In particular, discontinuity in tangential velocity along a stream surface is not allowed. This condition is true for a viscous flow and is imposed for an inviscid flow.
} 
Instead of doing this in section 2, we shall recast Weiss's formula for an irrotational flow as a simple formula for the image of the radial velocity component and then use the latter to derive the image of a rotational flow due to the sphere, a potential flow defined by a triple integral of $\boldsymbol{\Omega}$. We then use the asymptotic matching procedure detailed in $[4,5]$ to derive the expression for the far-field sound generated by the vorticity field in the presence of the sphere. In section 3 we apply the formulas to define the velocity of a slender filament outside a sphere and the far-field sound. Comparison of the current analysis with that of Lighthill [6] for a vortex element is then presented. As a consequence of the comparison, we derive in the appendix an alternate formula for the vortical flow, showing that the velocity field is defined by only two components of vorticity, say the transverse vorticity vector.

2. Analysis. Let $(u, v, w)$ denote the three components of velocity in spherical coordinates. From Weiss's formula (2) we get the image radial velocity

$$
u^{*}(r, \theta, \phi)=\partial_{r} \Phi^{*}=-\left[a^{3} / r^{3}\right] u_{0}(\bar{r}, \theta, \phi),
$$

where $\bar{r}=a^{2} / r$ is the mirror image of $r$. Since $\bar{r}<a$ when $r>a$, (8) says that

$\{5\}$ the image radial velocity $u^{*}$ outside of the sphere is equal to $-a^{3} / r^{3}$ times the original radial velocity $u_{0}$ at the mirror image point inside the sphere.

Now we identify $u^{*}$ as the radial velocity of potential $\Phi^{*}$, i.e., $u^{*}(r, \theta, \phi)=$ $\partial_{r} \Phi^{*}(r, \theta, \phi)$, and obtain

$$
\Phi^{*}(r, \theta, \phi)=\frac{1}{a} \int_{0}^{a^{2} / r} \bar{r}^{\prime} \partial_{\bar{r}^{\prime}} \Phi_{0}\left(\bar{r}^{\prime}, \theta, \phi\right) d \bar{r}^{\prime}
$$

where $\bar{r}^{\prime}=a^{2} / r^{\prime}$. Thus (9) is equivalent to (2). Consequently, we have that

$\{6\}$ the image radial velocity $u^{*}$ defined by (8) represents the radial velocity of a potential flow $\Phi^{*}$ outside of the sphere $\mathcal{S}$, fulfilling the requirements $\{1\}$, $\{2\}$, and $\{3\}$.

The rotational flow field $\mathbf{v}$ in the space that we are considering is regular and irrotational inside the sphere $\mathcal{S}$, because of (4), and hence the formula for the image radial velocity (8) remains applicable.

First we use (6) to relate the radial component of $\mathbf{v}$ to the vorticity field

$$
u(r, \theta, \phi)=\frac{1}{4 \pi} \iiint_{R \geq a} \frac{\hat{r} \cdot \mathbf{y} \times \mathbf{\Upsilon}(\mathbf{y})}{|\mathbf{x}-\mathbf{y}|^{3}} d \mathcal{V}
$$

where $\mathbf{\Upsilon}$ denotes the transverse vorticity vector

$$
\mathbf{\Upsilon}(\mathbf{y})=\mathbf{\Omega}-(\boldsymbol{\Omega} \cdot \hat{\mathbf{y}}) \hat{\mathbf{y}}
$$

Here we made use of the fact that $\hat{r}(\theta, \phi)$ is the unit vector of $\mathbf{x}$ and the radial vorticity vector $(\boldsymbol{\Omega} \cdot \hat{\mathbf{y}}) \hat{\mathbf{y}}$ is parallel to $\hat{\mathbf{y}}$, the unit vector of $\mathbf{y}$. In (10), the dependence of $u$ on $r$ appears implicitly via the distance

$$
|\mathbf{x}-\mathbf{y}|=\left[R^{2}-2 r R \mu+r^{2}\right]^{1 / 2},
$$

where $R=|\mathbf{y}|, \mu(\theta, \phi)=\cos \beta=\hat{r}(\theta, \phi) \cdot \hat{\mathbf{y}}$, and $\beta$ denotes the angle between the position vectors $\mathbf{x}$ and $\mathbf{y}$.

With $\bar{r}=a^{2} / r$, we have $\bar{r}<a$ for $r>a$ and the point $\mathbf{x}^{*}=\bar{r} \hat{r}$ inside $\mathcal{S}$ is the spherical mirror image of the point $\mathbf{x}$ outside. We then apply (8) or rule $\{5\}$ to define 
the radial component of the image velocity $\mathbf{v}^{*}$ outside of $\mathcal{S}$. The result is

$$
u^{*}(r, \theta, \phi)=-\frac{1}{4 \pi} \iiint_{R \geq a} \frac{\lambda^{3}}{a^{3} Z^{3}(\lambda, \mu)} \hat{r}(\theta, \phi) \cdot \mathbf{y} \times \mathbf{\Upsilon}(\mathbf{y}) d \mathcal{V},
$$

where

$$
Z(\lambda, \mu)=\left[1-2 \lambda \mu+\lambda^{2}\right]^{1 / 2} \quad \text { and } \quad \lambda=\frac{\bar{r}}{R}=\frac{a^{2}}{r R} .
$$

Using (9), the corresponding image potential outside $\mathcal{S}$ is ${ }^{2}$

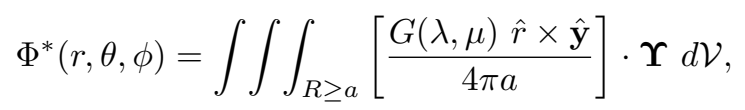

where

$$
G(\lambda, \mu)=\int_{0}^{\lambda} \frac{\lambda^{\prime} d \lambda^{\prime}}{Z^{3}\left(\lambda^{\prime}, \mu\right)}=\frac{\lambda^{2}}{Z[Z+1-\lambda \mu]} .
$$

Note that $G$ is an elementary function of $\lambda$ and $\mu$ that depends only on $R=|\mathbf{y}|$, $r=|\mathbf{x}|$, and the angle $\beta$ between these two position vectors. Thus, the image potential outside $\mathcal{S}$ is a volume integral over the support of the vorticity field with integrand regular. From (15), we observe that

$\{7\}$ the image potential $\Phi^{*}$ is independent of the radial vorticity component. In the integrand of the volume integral in (15), the transverse component of the vector inside the square bracket can be interpreted as the image potential of a vortex element of unit strength induced by the sphere.

The dependence of $\Phi^{*}$ on the two spherical coordinates $\theta, \phi$ of $\mathbf{x}$ appears only through $\hat{r}$, which is seen explicitly in the integrand and implicitly via $\mu$ in $G$, while the dependence on $r$ appears only through $\lambda$ in G. We can compute the $\theta$ - and $\phi$-components of the image velocity from the components of $\nabla \Phi^{*}$. These two components together with the radial component, $u^{*}$ in (13), define the image velocity $\mathbf{v}^{*}$ regular outside the rigid sphere. However, the vortical flow $\mathbf{v}$ in $\Re^{3}$ is usually expressed in Cartesian coordinates. To obtain the resultant velocity $\mathbf{V}=\mathbf{v}+\mathbf{v}^{*}$, we derive equations for the Cartesian components of the image velocity, i.e., $\nabla \Phi^{*}$,

$$
v_{j}^{*}=\frac{-1}{4 \pi a} \iiint_{R \geq a} d \mathcal{V}\left\{G \partial_{x_{j}} \hat{r}+\left[\partial_{\lambda} G \partial_{x_{j}} \lambda+\partial_{\mu} G \partial_{x_{j}} \mu\right] \hat{r}\right\} \cdot[\hat{\mathbf{y}} \times \mathbf{\Omega}(\mathbf{y})]
$$

for $j=1,2,3$, where

$$
\begin{aligned}
& \partial_{\lambda} G=\lambda / Z^{3}(\lambda, \mu), \quad \partial_{x_{j}} \lambda=\frac{-a^{2} x_{j}}{R r^{3}}, \\
& \partial_{\mu} G=\frac{\lambda^{3}\left[Z^{2}+2 Z+1-\lambda \mu\right]}{Z\left[Z^{2}+Z(1-\lambda \mu)\right]^{2}}, \\
& \partial_{x_{j}} \hat{r}=\partial_{x_{j}} \frac{\mathbf{x}}{r}=\frac{\hat{\jmath}}{r}-\frac{x_{j} \mathbf{x}}{r^{3}}, \quad \text { and } \quad \partial_{x_{j}} \mu=\hat{\mathbf{y}} \cdot \partial_{x_{j}} \hat{r} .
\end{aligned}
$$

\footnotetext{
${ }^{2}$ We use $\mathbf{y} \times \boldsymbol{\Upsilon}$ instead of $\mathbf{y} \times \boldsymbol{\Omega}$ to emphasize the fact that the vector product is independent of the radial component of $\boldsymbol{\Omega}$. In the evaluation of the volume integral, we shall use $\hat{\mathbf{y}} \times \boldsymbol{\Omega}$ or $\hat{\mathbf{y}} \times \mathbf{\Upsilon}$ when $\boldsymbol{\Omega}$ or $\boldsymbol{\Upsilon}$ is prescribed. This will also be applied to the vector product appearing in the volume integrals later.
} 
To obtain the far-field sound induced by the image potential, we need the farfield behavior of $\Phi^{*}$, i.e., where $r / a \gg 1$ and show its dependence on time $t$. With $\lambda=a^{2} /(r R)=O\left(r^{-1}\right)$ and $\mu=\hat{r} \cdot \hat{\mathbf{y}}$, we have in the far field

$$
Z=1-\lambda \mu+O\left(r^{-2}\right), \quad G=\lambda^{2}\left[1+2 \lambda \mu+O\left(r^{-2}\right)\right] / 2,
$$

and the far-field image potential

$$
\begin{aligned}
\Phi^{*}(t, \mathbf{x})= & \frac{1}{8 \pi r^{2}} \iiint_{R \geq a} d \mathcal{V} \hat{r} \cdot \mathbf{y} \times \boldsymbol{\Omega}(t, \mathbf{y})\left[\frac{a}{R}\right]^{3} \\
& +\frac{1}{4 \pi r^{3}} \iiint_{R \geq a} d \mathcal{V} \hat{r} \cdot \mathbf{y} \times \boldsymbol{\Omega}(t, \mathbf{y})[\hat{r} \cdot \mathbf{y}]\left[\frac{a}{R}\right]^{5}+O\left(r^{-4}\right) \\
= & \frac{-1}{4 \pi} \sum_{i=1}^{3} \partial_{x_{i}}\left[\frac{D *_{i}(t)}{r}\right]+\frac{1}{12 \pi} \sum_{i=1}^{3} \sum_{l=1}^{3} \partial_{x_{i}} \partial_{x_{l}}\left[\frac{q_{i l}^{*}(t)}{r}\right]+O\left(r^{-4}\right) .
\end{aligned}
$$

The first term represents doublets with strengths

$$
D_{i}^{*}(t)=\frac{1}{2} \iiint_{R \geq a} d \mathcal{V}\left[y_{j} \omega_{k}-y_{k} \omega_{j}\right]\left(\frac{a}{R}\right)^{3}
$$

for $i=1,2,3$ and $i, j, k$ in cyclic order. Here $y_{i}$ and $\omega_{i}$ denote the $i$ th component of $\mathbf{y}$ and $\boldsymbol{\Omega}(t, \mathbf{y})$, respectively. The strength $D_{i}^{*}(t)$ is a first moment of vorticity weighted by $[a / R]^{3}$.

The second term in (20) represents quadrupoles with components

$$
q_{i l}^{*}=\iiint_{R \geq a} d \mathcal{V}\left[y_{j} \omega_{k}-y_{k} \omega_{j}\right] y_{l}\left(\frac{a}{R}\right)^{5}
$$

for $i, l=1,2,3$ and $i, j, k$ in cyclic order. The strength $q_{i l}^{*}(t)$ is a second moment of $\boldsymbol{\Omega}$ weighted by $(a / R)^{5}$.

The dependence of the doublet and quadrupole strengths, $D_{i}^{*}$ and $q_{i l}^{*}$, on $t$ is defined by the solution of the vorticity evolution equation outside the sphere with convective velocity $\mathbf{v}+\mathbf{v}^{*}$.

By matching the far-field solution of the image potential in $\mathbf{x}$ to the outer solution of the acoustic field in the stretched variable $\tilde{r}=M r$ or $\tilde{\mathbf{x}}=M \mathbf{x}$, we obtain the acoustic pressure induced by the image potential $\Phi^{*}$,

$$
\begin{aligned}
\tilde{p}^{*} & =-\rho \partial_{t} \tilde{\Phi}^{*}(t, \tilde{\mathbf{x}}) \\
& =\left[\frac{\rho}{4 \pi}\right]\left\{M^{2} \sum_{i=1}^{3} \partial_{\tilde{x}_{i}}\left[\frac{\dot{D}_{i}^{*}(\tau)}{\tilde{r}}\right]-\frac{M^{3}}{3} \sum_{i=1}^{3} \sum_{l=1}^{3} \partial_{\tilde{x}_{i} \tilde{x}_{l}}^{2}\left[\frac{\dot{q}_{i l}^{*}(\tau)}{\tilde{r}}\right]+O\left(M^{4}\right)\right\},
\end{aligned}
$$

where $\tau=t-r / C=t-\tilde{r} /(M C)$ denotes the retarded time, $\rho$ is the ambient density, and $M \ll 1$ is a typical Mach number of the vortical flow. The leading term in (23) is $O\left(M^{2}\right)$ representing dipoles, and the second one is $O\left(M^{3}\right)$ representing quadrupoles.

In the absence of the sphere, the resultant far-field doublet strength of a vortical flow is given by the first moment of vorticity in $\Re^{3}$. The first moment was shown to be time invariant by Moreau [7] using the first moment of the vorticity evolution equation. Thus the far-field doublet strength is independent of $t$ and the corresponding dipole sound vanishes. The leading term in the acoustic field is $O\left(M^{3}\right)$ representing 
quadrupoles $[4,5]$. Now in the presence of a rigid sphere, Moreau's theorem is no longer applicable. The first moment of vorticity $\boldsymbol{\Omega}$ is time dependent because the vorticity field is now convected by the image velocity $\mathbf{v}^{*}$ in addition to $\mathbf{v}$. Furthermore, the contribution of the image potential to the doublet is defined by the weighted vorticity first moment (21). Both the unsteady vector potential $\mathbf{A}$ induced by the vorticity field and the image potential $\Phi^{*}$ contribute to dipole sound. It follows that

$\{8\}$ the acoustic field induced by a vortical flow in the presence of the sphere is one order stronger than that in absence of the sphere.

We now point out the effect of a shift of origin by $O(\ell)$ on the far-field sound. The effect appears in the change of $r=|\mathbf{x}|$ by $O(\ell / r)$ and in the strengths of the dipoles and quadrupoles.

For a vortical flow in $\Re^{3}$, the dipole strength, i.e., the first moment of vorticity, is independent of $t$ and also of the shift of origin. This is true because the volume integral of vorticity is zero. A shift of origin will change the strengths of quadrupoles, i.e., the second moments of vorticity, by $O(1)$. But these changes are independent of $t$ because the first moments are time invariant. Thus, a shift of origin will change the acoustic pressure prediction by only $O(\ell / r)=O(M \ell / \tilde{r})$.

For a vortical flow outside a rigid body, e.g., a sphere, the image solution (15) and hence the convective velocity depend on the choice of the origin. Consequently, a shift of origin by $O(\ell)$ will change the strengths of the far-field dipoles and quadrupoles of the vortical field plus the image potential flow by $O(1)$. Thus for the computation of the strengths, the origin has to coincide with that for the vortical flow, i.e., the center of the sphere.

We also note that the above representation of the acoustic dipole in terms of the first moment of vorticity provides an attractive alternative to the classical results of Powell [8], in which the acoustic dipole is expressed in terms of an area integral over the surface distribution of Bernoulli pressure. In particular, the present formulation avoids the need to perform delicate and time-consuming determination of the Bernoulli pressure, which generally requires inverting a pressure Poisson equation or integrating the momentum equation along solid surfaces.

In the next section, we apply the formulas for the image velocity, potential, and acoustic field, (10) to (23), to the special case that the vorticity is highly concentrated in the core of a slender vortex filament.

3. A slender vortex filament outside a sphere. Consider the vector potential $\mathbf{A}$ induced by a slender vortex filament of strength or circulation $\Gamma$. Let $\mathbf{X}(s)$ denote the position vector of the centerline $\mathcal{C}$ of a slender vortex filament of length $\mathrm{L}$ and $s$ denote the arc length of $\mathcal{C}$ (at instant $t$ ). For a point $\mathbf{x}$ at a distance from $\mathcal{C}$ much larger than the core size $\delta$ the leading-order velocity term in (6) is given by the line Biot-Savart integral

$$
\mathbf{v}(t, \mathbf{x})=-\frac{\Gamma}{4 \pi} \int_{\mathcal{C}} \frac{(\mathbf{x}-\mathbf{X}) \times \hat{\tau} d s}{|\mathbf{x}-\mathbf{X}|^{3}},
$$

where $|\mathbf{x}-\mathbf{X}|=O(1) \gg \delta, d \mathbf{X}(s)=\hat{\tau}(s) d s$, and $\hat{\tau}(s)$ denotes the unit tangent vector of $\mathcal{C}$ at $\mathbf{X}(s)$. The volume integral over the support of $\boldsymbol{\Omega}$ in (6) is reduced to the line integral along $\mathcal{C}$ in (24) by the following approximation rule:

$\{9\}$ Replace $\mathbf{y}$ by $\mathbf{X}$, the vortex volume element $\boldsymbol{\Omega}(\mathbf{y}) d \mathcal{V}$ by the vortex line element $\Gamma d \mathbf{X}(s)$.

We assume that the filament lies outside of the sphere $r=a$, with $a=O(1)$, and the distance between the filament and the sphere is much larger than the typical 
vortical core size $\delta$ and the boundary layer thickness around the sphere. Thus, we have

$$
|\mathbf{X}(s)|>a \text { for } 0 \leq s \leq L .
$$

This condition is consistent with (4); i.e., the support of $\boldsymbol{\Omega}$ lies outside the sphere.

By omitting the boundary layer, i.e., assuming the Reynolds number $R_{e} \gg 1$, the formulas derived in the preceding section for the image velocity, potential, and far-field sound are applicable. Using the rule of approximations $\{9\}$, we identify $\hat{\mathbf{y}}$ as $\hat{\mathbf{X}}, R=|\mathbf{y}|$ as $R=|\mathbf{X}|$, and $\mu=\cos \beta=\hat{r} \cdot \hat{\mathbf{X}}$ and keep the same definition for $\bar{r}=A^{2} / r$ and the definitions of $\lambda$ and $Z(\lambda, \mu)$ by (14). Equation (15) for the image potential becomes

$$
\Phi^{*}(\mathbf{x})=\int_{\mathcal{C}}\left[\frac{G(\lambda, \mu) \hat{r} \times \hat{\mathbf{X}}(s)}{4 \pi a}\right] \cdot \hat{\tau}(s) \Gamma d s .
$$

Thus, the image potential $\Phi^{*}(\mathbf{x})$ outside the sphere is a line integral along $\mathcal{C}$ with integrand regular. From (26), we arrive at the following observation corresponding to $\{7\}$ :

$\{10\}$ In the integrand of the line integral for the image potential $\Phi^{*},(26)$, the tangential component of the vector inside the square bracket can be interpreted as the image potential of a tangential vortex element of unit strength induced by the sphere.

Formula (17) for the components of the image velocity $\mathbf{v}^{*}$ becomes

$$
v_{j}^{*}=\frac{-\Gamma}{4 \pi a} \int_{\mathcal{C}}\left\{G \partial_{x_{j}} \hat{r}+\left[\partial_{\lambda} G \partial_{x_{j}} \lambda+\partial_{\mu} G \partial_{x_{j}} \mu\right] \hat{r}\right\} \cdot[\hat{\tau}(s) \times \hat{\mathbf{X}}(s)] d s
$$

for $j=1,2,3$. The three partial derivatives in the integrand are defined by (18a), (18b), and (18c) with $\mathbf{y}$ replaced by $\mathbf{X}$. The image velocity is regular outside the sphere, in particular on the centerline $\mathcal{C}$; therefore the velocity of the filament in the presence of the sphere is

$$
\dot{\mathbf{X}}\left(s_{0}\right)=\mathbf{q}\left(s_{0}\right)+\mathbf{v}^{*}\left(\mathbf{X}\left(s_{0}\right)\right)
$$

where $\mathbf{q}$ denotes the velocity induced by the filament alone. It depends on the core structure and the finite part of the Biot-Savart integral [4]. The contribution of the image potential to the far-field pressure due to the presence of the sphere can be found from (23) using the following expressions for the strengths of doublets and quadrupoles

$$
D_{i}^{*}(t)=\frac{\Gamma}{2} \int_{\mathcal{C}} d s\left[X_{j} \tau_{k}-X_{k} \tau_{j}\right]\left(\frac{a}{|\mathbf{X}|}\right)^{3}
$$

and

$$
q_{i l}^{*}=\Gamma \int_{\mathcal{C}} d s\left[X_{j} \tau_{k}-X_{k} \tau_{j}\right] X_{l}\left(\frac{a}{|\mathbf{X}|}\right)^{5}
$$

In 1956, Lighthill [6] constructed the image of a given vortex element $\mathbf{J}$ located at $\mathbf{X}$ outside of the sphere, e.g., a segment of a vortex line $\mathcal{C}$, with $\mathbf{J}=\Gamma d \mathbf{X}(s)$ and $|\mathbf{X}|>a$, so that the resultant velocity $\mathbf{V}$ induced by the vortex element $\mathbf{J}$ outside 
and the image vorticity $\mathbf{W}$ inside the sphere has zero radial component on the sphere. It was duly noted that a radial vorticity vector does not induce radial velocity on the sphere. The radial velocity induced by the transverse vortex element outside of the sphere can only be cancelled by an image transverse element scaled by $-a / R$ located at the inverse point $a^{2} \mathbf{X} / R^{2}$ with $R=|\mathbf{X}|$. To make the image vorticity field inside the sphere divergence-free, an image radial vortex element at the inverse point and a radial line vortex extending from the inverse point to the origin are needed. The major effort of [6] is devoted to applying the divergence-free condition to a distributed vorticity field and then interpreting the result for the vortex element by physical reasoning.

When the vorticity field $\boldsymbol{\Omega}$ outside the sphere is a vortex line $\mathcal{C}$, the image vorticity field $\mathbf{W}$ inside the sphere is composed of a line distribution of vorticity along the image of $\mathcal{C}$ plus a conical vortex surface with the origin as its vertex and the image of $\mathcal{C}$ as the baseline. However, the velocity $\mathbf{V}$ induced by the combined vorticity field $\boldsymbol{\Omega}+\mathbf{W}$ in $\Re^{3}$ can be expressed as a line integral along $\mathcal{C}$ and the "algebraic complexity of the integrand is not increased by a factor more than three over that of the Biot-Savart integral for $\boldsymbol{\Omega}$ alone." The formulas for the three Cartesian components of $\mathbf{V}$ in [6] are in agreement with the formulas (17) given above. The formula coming from (15) shows that the additional or image velocity $\mathbf{v}^{*}$ due to the sphere depends only on the transverse vorticity field outside of the sphere. The derivation presented here is straightforward because it makes use of the fact that the image velocity $\mathbf{v}^{*}$ outside of the sphere is irrotational and Weiss's theorem [1] is recast as equation (8) relating the radial component $u^{*}$ of $\mathbf{v}^{*}$ directly to that of $\mathbf{v}$ inside the sphere, where $\mathbf{v}$ is irrotational. From the image radial component $u^{*}$, we obtain the image potential and the corresponding acoustic potential and pressure.

In hindsight, we could say that the determination of the relatively complex radial image vorticity field, making the vorticity field $\mathbf{W}$ divergence-free, is not needed to define the additional velocity $\mathbf{v}^{*}$. It is needed [6] when we apply the formula (6) to define the velocity induced by the vorticity field or the Biot-Savart formula for a vortex line. In general, one can modify the formula so that the velocity is defined by only two components of the vorticity field, as shown in the appendix.

We also note that we can use Lighthill's formula for the velocity $\mathbf{V}$ induced by the vorticity field $\boldsymbol{\Omega}+\mathbf{W}$ in $\Re^{3}$, with the rigid sphere replaced by the image $\mathbf{W}$ inside the sphere. We can then derive the corresponding acoustic potential, $\tilde{\Phi}$ at instant $t$, and find that the leading terms are dipoles with strengths given by the first moments of $\boldsymbol{\Omega}+\mathbf{W}$ over $\Re^{3}$ and are equivalent to those defined above. The vorticity evolution equation with convective velocity $\mathbf{V}$ defines the temporal variation of the vorticity field $\boldsymbol{\Omega}$ outside of the sphere while the image field $\mathbf{W}$ inside the sphere at each instant is given by Lighthill's formula. Thus we cannot apply Moreau's theorem to the first moments $\boldsymbol{\Omega}+\mathbf{W}$ in $\Re^{3}$ and cannot conclude that the dipole strengths are time-invariant, producing zero acoustic pressure.

4. Conclusion. We study the flow field $\mathbf{V}(t, \mathbf{x})$ induced by a vorticity field $\boldsymbol{\Omega}(t, \mathbf{x})$ outside a sphere $|\mathbf{x}|=a$. We write $\mathbf{V}=\mathbf{v}+\mathbf{v}^{*}$, where $\mathbf{v}$ represents the vortical flow induced by $\boldsymbol{\Omega}$ in $\Re^{3}$ without the sphere while $\mathbf{v}^{*}$ represents the additional or image flow due to the presence of the sphere. At each instant, $\mathbf{v}$ is related to $\boldsymbol{\Omega}$ by (6), via the vector potential $\mathbf{A}$ given by the Poisson integral of $\boldsymbol{\Omega}$. The image velocity is a potential flow with radial velocity on the sphere cancelling that of $\mathbf{v}$. The image potential $\Phi^{*}$ is related directly to $\boldsymbol{\Omega}$ by (15). To derive this formula we note that the support of $\boldsymbol{\Omega}$ is outside the sphere. Therefore, $\mathbf{v}$ is irrotational and Weiss's 
theorem, which defines the image of a potential flow due to a sphere, is applicable. To simplify the derivation of (15), we recast Weiss's theorem as equation (8) relating the radial image velocity $u^{*}$ at a point $\mathbf{x}$ outside the sphere to the radial component of $\mathbf{V}$ at the image point of $\mathbf{x}$ inside the sphere. Using (8) for $u^{*}$ or $\partial_{r} \Phi^{*}$ we arrive at (15) and (17) for the image potential $\Phi^{*}$ and velocity $\mathbf{v}^{*}$. The evolution of the vortical field is now governed by the vorticity transport equation with the convective velocity $\mathbf{v}+\mathbf{v}^{*}$ related to $\boldsymbol{\Omega}$ by the integral equations (8) and (17). From the far-field behavior of $\Phi^{*}(t, \mathbf{x})$ we obtain the acoustic potential $\tilde{\Phi}^{*}$ and then (23) for the contribution of the image potential to the far-field sound. Evaluation of this contribution requires the time derivative of $\boldsymbol{\Omega}$ and, hence, the solution of the vorticity evolution equation. It is shown that the presence of the sphere induces dipole and quadrupole sound; in contrast, a vortical flow $\mathbf{v}$ in $\Re^{3}$ radiates quadrupole sound only.

Formulas derived in section 2 are applied in section 3 to define the velocity of a slender vortex filament and far-field sound due to the motion of the filament in the presence of a sphere.

Appendix. An alternate formula for the vortical flow. This representation is applicable to a more general setting, i.e., to a divergence-free vector field $\mathbf{v}(\mathbf{x})$ when $\nabla \times \mathbf{v}=\boldsymbol{\Omega}(\mathbf{y})$ is specified. Here we shall assume a weaker far-field condition on $\boldsymbol{\Omega}$

$$
\boldsymbol{\Omega}=O\left(|\mathbf{x}|^{-3}\right) \text { as } \quad|\mathbf{x}| \rightarrow \infty,
$$

instead of the stronger condition of exponential decay in $|\mathbf{x}|$ or of bounded support. The standard formula for $\mathbf{v}$ is

$$
\mathbf{v}(\mathbf{x})=\frac{-1}{4 \pi} \iiint_{\Re^{3}} d \mathcal{V} \frac{(\mathbf{x}-\mathbf{y}) \times \boldsymbol{\Omega}(\mathbf{y})}{|\mathbf{x}-\mathbf{y}|^{3}} .
$$

Since $\boldsymbol{\Omega}$ is divergence-free, we need only to specify two components of $\boldsymbol{\Omega}$ and use the third component to fulfill the divergence-free condition. We shall derive a formula relating the velocity to two vorticity components and, hence, eliminate the need of solving for the third component.

Here we consider the vector field in spherical coordinates $r, \theta, \phi$, with $\mathbf{x}=r \hat{r}(\theta, \phi)$ and denote the three components of $\boldsymbol{\Omega}$ by $\xi, \eta$, and $\zeta$, i.e.,

$$
\boldsymbol{\Omega}=\xi \hat{r}+\boldsymbol{\Upsilon}=\xi \hat{r}+\eta \hat{\theta}+\zeta \hat{\phi} .
$$

Here $\Upsilon$ denotes the transverse vector. When the transverse vector or the two components, $\eta$ and $\zeta$, of $\boldsymbol{\Omega}$ are specified, the radial component $\xi$ is defined by the divergencefree condition

$$
\sin \theta \partial_{r}\left(r^{2} \xi\right)+r \partial_{\theta}(\eta \sin \theta)+r \partial_{\phi} \zeta=0
$$

and the far-field condition. To derive a formula for $\mathbf{v}$ depending only on $\mathbf{\Upsilon}$, we first decompose the triple integral in (A2) into two integrals, involving the radial and transverse vectors of $\boldsymbol{\Omega}$, respectively. The result is

$$
\begin{aligned}
\mathbf{v}(\mathbf{x})= & \frac{-\mathbf{x}}{4 \pi} \times \int_{0}^{2 \pi} \int_{0}^{\pi} \sin \theta^{\prime} d \theta^{\prime} d \phi^{\prime} \int_{0}^{\infty} R^{2} d R \frac{\hat{\mathbf{y}} \xi(\mathbf{y})}{|\mathbf{x}-\mathbf{y}|^{3}} \\
& -\frac{1}{4 \pi} \int_{0}^{2 \pi} \int_{0}^{\pi} \sin \theta^{\prime} d \theta^{\prime} d \phi^{\prime} \int_{0}^{\infty} R^{2} d R \frac{(\mathbf{x}-\mathbf{y}) \times \mathbf{\Upsilon}(\mathbf{y})}{|\mathbf{x}-\mathbf{y}|^{3}}
\end{aligned}
$$


with $\mathbf{y}=R \hat{\mathbf{y}}\left(\theta^{\prime}, \phi^{\prime}\right)$. In terms of the spherical coordinates, the distance is

$$
|\mathbf{x}-\mathbf{y}|=\left[r^{2}+(R)^{2}+2 r R \mu\right]^{1 / 2}=\left[\left(1-\mu^{2}\right) r^{2}+(R-r \mu)^{2}\right]^{1 / 2},
$$

where $\mu=\hat{r} \cdot \hat{\mathbf{y}}=\cos \theta \cos \theta^{\prime}+\sin \theta \sin \theta^{\prime} \cos \left(\phi-\phi^{\prime}\right)$. Note that the radial vorticity appears only in the first triple integral in (A3) and that $\mu$ and $\hat{\mathbf{y}}$ depend only on $\theta^{\prime}$ and $\phi^{\prime}$ but not on $R$. We then carry out integration by parts in $d R$ to replace the first triple integral by

$$
\frac{\mathbf{x}}{4 \pi} \times \int_{0}^{2 \pi} \int_{0}^{\pi} \sin \theta^{\prime} d \theta^{\prime} d \phi^{\prime} \hat{\mathbf{y}} \int_{0}^{\infty} \frac{R-r \mu}{|\mathbf{x}-\mathbf{y}| r^{2}\left(1-\mu^{2}\right)}\left[\partial_{R}\left(R^{2} \xi\right)\right] d R .
$$

Now we use the divergence-free condition to replace the term $\partial_{R}\left(R^{2} \xi\right)$ in the integrand above and rewrite (A5) as

$$
\begin{aligned}
\mathbf{v}(\mathbf{x})= & \frac{-\mathbf{x}}{4 \pi r^{2}} \times \int_{0}^{2 \pi} \int_{0}^{\pi} \hat{\mathbf{y}} d \theta^{\prime} d \phi^{\prime} \int_{0}^{\infty} R d R \frac{\left[\partial_{\theta^{\prime}}\left(\eta \sin \theta^{\prime}\right)+\partial_{\phi^{\prime}} \zeta\right](R-r \mu)}{|\mathbf{x}-\mathbf{y}|\left(1-\mu^{2}\right)} \\
& -\frac{1}{4 \pi} \int_{0}^{2 \pi} \int_{0}^{\pi} \sin \theta^{\prime} d \theta^{\prime} d \phi^{\prime} \int_{0}^{\infty} R^{2} d R \frac{(\mathbf{x}-\mathbf{y}) \times \mathbf{\Upsilon}(\mathbf{y})}{|\mathbf{x}-\mathbf{y}|^{3}}
\end{aligned}
$$

Thus, we arrive at an equation for the vector field $\mathbf{v}$ depending only on the two transverse components of $\boldsymbol{\Omega}$. In principle, we can remove the $\theta^{\prime}$ - and $\phi^{\prime}$-derivatives of the transverse components in the first triple integral by integration by parts, but we shall not do it here because it may be easier to carry out the numerical integration in the present form than the new one.

At any rate, it is clear that we can derive an alternate formula for $\mathbf{v}$ in terms of two components of $\boldsymbol{\Omega}$ in either Cartesian coordinates or cylindrical coordinates.

\section{REFERENCES}

[1] P. Weiss, On hydrodynamical images - Arbitrary irrotational flow disturbed by a sphere, Proc. Cambridge Phil. Soc., 40 (1944), pp. 259-261.

[2] J. B. Keller And S. I. Rubinow, Force on a rigid sphere in an incompressible fluid, Phys. Fluids, 14 (1971), pp. 1302-1304.

[3] H. LAmb, Hydrodynamics, Cambridge University Press, 1945.

[4] L. Ting And R. Klein, Viscous Vortical Flows, Lecture Notes in Physics 374, Springer-Verlag, New York, 1991

[5] L. Ting And M. J. Miksis, On vortical flow and sound generation, SIAM J. Appl. Math., 50 (1990), pp. 521-536.

[6] M. J. Lighthill, The image system of a vortex element in a rigid sphere, Proc. Cambr. Philos. Soc., 52 (1956), pp. 317-321.

[7] J. J. Moreau, Sur deux theoremes generaux de la dynamique d'un milieu incompressible illimite, C. R. Acad. Sci. Paris, 229 (1948), pp. 1420-1422.

[8] A. Powell, Theory of vortex sound, J. Acoust. Soc. Amer., 36 (1964), pp. 177-195. 